\title{
Analysis of delayed convergence in the three-noded Timoshenko beam element using the function space approach
}

\author{
SOMENATH MUKHERJEE ${ }^{1}$ and GANGAN PRATHAP ${ }^{2 *}$ \\ ${ }^{1}$ Structures Division, National Aerospace Laboratories, Bangalore 560 017, India \\ ${ }^{2}$ Centre for Mathematical Modelling and Computer Simulation, \\ Bangalore 560 037, India \\ e-mail: $\{$ somu, gp $\} @$ cmmacs.ernet.in
}

MS received 30 March 2002; revised 21 June 2002

\begin{abstract}
Despite satisfying only completeness and continuity requirements, elements often perform erroneously in a certain class of problems, called the locking situations, where they display spurious stress oscillations and enhanced stiffness properties. The function space approach that effectively substantiates the postulates of the field consistency paradigm is an efficient tool to reveal the fundamental cause of locking phenomena, and propose methods to eliminate this pathological problem. In this paper, we review the delayed convergence behaviour of three-noded Timoshenko beam elements using the rigorous function space approach. Explicit, closed form algebraic results for the element strains, stresses and errors have been derived using this method. The performance of the field-inconsistent three-noded Timoshenko beam element is compared with that of the field-inconsistent twonoded beam element. It is demonstrated that while the field-inconsistent two-noded linear element is prone to shear locking, the field-inconsistent three-noded element is not very vulnerable to this pathological problem, despite the resulting shear oscillations.
\end{abstract}

Keywords. Strain projection; field-inconsistent/consistent function subspaces; variational correctness; shear locking; delayed convergence; spurious shear oscillations.

\section{Introduction}

It is well-known that isoparametric Timsoshenko beam elements, in general, display enhanced stiffness properties and spurious stress oscillations, despite satisfying completeness and continuity requirements (Zienkiewicz \& Taylor 1991; Prathap 1993). Various explanations have

\footnotetext{
*For correspondence

A list of symbols is given at the end of the paper
} 
been offered for the origin of these pathological symptoms that are associated with a phenomenon known as locking. It has been argued (Tessler \& Hughes 1983) that locking is caused by ill-conditioning of the stiffness matrix due to the very large magnitude of the shear stiffness terms as compared to the those of bending stiffness. Carpenter et al (1986) have shown that locking occurs due to coupling between the shear deformation and bending deformation, and that it can be eliminated by adopting strain fields such that these are appropriately decoupled. Prathap $(1982,1987)$ has shown that elements lock because they inadvertently enforce spurious constraints that arise from inconsistencies in the strains developed from the assumed displacement functions. Using the two-noded Timoshenko beam element as illustration, Mukherjee \& Prathap (2001) have recently shown how locking manifests itself in low order elements, and proposed methods to predict and eliminate locking, using the function space approach.

In this paper, we address the characteristic features of delayed convergence phenomenon in higher order elements like the three-noded Timoshenko beam element using the mathematically rigorous function space approach that unifies the arguments forwarded by Carpenter $e t$ al (1986) with the field consistency paradigm of Prathap. Mild locking behaviour and delayed convergence in the three-noded beam element have earlier been observed, and explained by Prathap using the field consistency paradigm. For completeness, we first review the principles, based on the function space approach, behind locking phenomena.

\section{Function space analysis of strain projections under field inconsistency}

\subsection{Strain projections in finite element analysis}

For conservative systems, finite element analysis involves normal equations of the following form in an element (Zienkiewicz \& Taylor 1991),

$$
\int_{\text {ele }}[B]^{T}[D][B] \mathrm{d} x\left\{\delta^{e}\right\}=\int_{\text {ele }}[B]^{T}[D]\{\varepsilon\} \mathrm{d} x,
$$

where $[D]$ is the symmetric, positive definite, rigidity matrix and $\{\varepsilon\}$ is the true (analytical) strain. Here the element nodal displacement vector is $\left\{\delta^{e}\right\}$. The finite element strain vector (of $r$-rows i.e. of $r$ components), expressed as

$$
\{\varepsilon\}=[B]\left\{\delta^{e}\right\}
$$

is given as the orthogonal projection (Mukherjee \& Prathap 2001) of the analytical strain vector $\{\varepsilon\}$ onto the subspace $\mathfrak{B}$ that arises out of the strain-displacement matrix $[B]$, as

$$
\{\bar{\varepsilon}\}=\sum_{j=1}^{m} \frac{\left\langle u_{j}, \varepsilon\right\rangle}{\left\langle u_{j}, u_{j}\right\rangle}\left\{u_{j}\right\},
$$

where the vectors $\left\{u_{i}\right\},(i=1,2 . . m)$ represent the $m$-orthogonal basis vectors that span the $m$-dimensional subspace $\mathfrak{B}$, (i.e. $\left\langle u_{i}, u_{j}\right\rangle=0$ for $i \neq j$ ). The inner product of two vectors, $\{a\}$ and $\{b\}$, each of $r$ rows, is given here by

$$
\langle a, b\rangle=\int_{\text {ele }}\{a\}^{T}[D]\{b\} \mathrm{d} x .
$$


The size of the positive definite rigidity matrix $[D]$ is $r \times r$, and the integration in (4) is done over an element. The orthogonal basis vectors $\left\{u_{i}\right\}$ spanning the subspace $\mathfrak{B}$ can be determined by a standard procedure of linear algebra (Edwards \& Penny 1988), known as the Gram-Schmidt algorithm, applied to the column vectors of the matrix $[B]$. These basis vectors can be arbitrarily normalized. A brief review of the Gram-Schmidt algorithm for determining the orthogonal basis vectors is presented in the Appendix.

If $\{q\}$ is the error in the element strain vector having $r$ components,

$$
\{q\}=\{\varepsilon\}-\{\bar{\varepsilon}\}
$$

then the error norm squared, also interpreted as the energy of the error, is given by

$$
\|q\|^{2}=\langle q, q\rangle=\int_{\text {ele }}\{q\}^{T}[D]\{q\} \mathrm{d} x .
$$

From the normal equation (1) we have the projection theorems (Edwards \& Penny 1988),

$$
\|q\|^{2}=\|\varepsilon\|^{2}-\|\bar{\varepsilon}\|^{2},
$$

i.e. $\quad$ the strain energy of the error = error in the strain energy.

It is also evident that

$$
\|\bar{\varepsilon}\|^{2}=\langle\bar{\varepsilon}, \varepsilon\rangle \text {. }
$$

A geometric analogy of the finite element strain vector $\{\bar{\varepsilon}\}$ as the orthogonal projection of the analytical strain vector $\{\varepsilon\}$ onto the $m$-dimensional $\mathfrak{B}$ subspace is presented in figure 1 . Here $m=$ total number of element degrees of freedom-total number of element rigid body motions. In general, for a strain vector involving $r$ components (i.e. $r$ rows), the $\mathfrak{B}$ space (arising out of the $[B]$ matrix of $r$ rows) of maximum degree $(n-1)$ of the parameter $\xi$, is a subspace of the $r \times n$ dimensional space $\mathfrak{P}_{n}^{r}(\xi)$ of ordered $r$-tuples of polynomials in $\xi$, denoted here by $\mathfrak{P}_{n}^{r}(\xi)$ upto degree $n-1$, bounded within the closed domain $(-1,1)$. The space $\mathfrak{P}_{n}^{r}(\xi)$ is represented by

$$
\mathfrak{P}_{n}^{r}=\left\{\{p\}:\{p\}=\sum_{i=1}^{n}\left\{\alpha_{i}\right\} \xi^{i-1},-1 \leq \xi \leq 1,\left\{\alpha_{i}\right\} \in R^{r}\right\} .
$$

Here $\Re^{r}$ is the $r$-dimensional space of real numbers. Standard orthogonal basis vectors, called the Legendre Orthogonals span the $r \times n$ dimensional space $\mathfrak{P}_{n}^{r}$ for a given degree $(n-1)$ of the polynomial in $\xi$. For instance, a system having a finite element strain vector of two

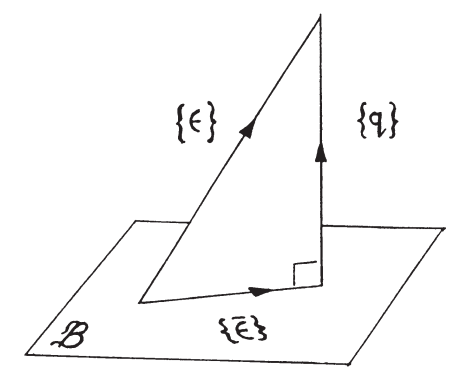

Figure 1. Geometric interpretation of the finite element strain vector as the orthogonal projection of the analytical strain vector onto a function subspace $\mathfrak{B}$ generated by the strain-displacement relationship. 
components with maximum degree of $\xi$ being one (linear in $\xi$ ), will generate a $\mathfrak{B}$ space that is a subspace of the four-dimensional space $\mathfrak{P}_{2}^{2}$ (linear in $\xi$ ). The standard basis vectors spanning the space $\mathfrak{P}_{2}^{2}$ are the Legendre Orthogonals, given by

$$
\left\{\mathscr{L}_{1}\right\}=[0,1]^{T}, \quad\left\{\mathscr{L}_{2}\right\}=[1,0]^{T}, \quad\left\{\mathscr{L}_{3}\right\}=[0, \xi]^{T}, \quad\left\{\mathscr{L}_{4}\right\}=[\xi, 0]^{T} .
$$

Similarly, for the six-dimensional space $\mathfrak{P}_{3}^{2}$ (quadratic in $\xi$ ), corresponding to a finite element strain vector of two components, or rows, with quadratic variation in $\xi$, the Legendre Orthogonals spanning it are

$$
\begin{aligned}
& \left\{\mathscr{L}_{1}\right\}=[0,1]^{T}, \quad\left\{\mathscr{L}_{2}\right\}=[1,0]^{T}, \quad\left\{\mathscr{L}_{3}\right\}=[0, \xi]^{T}, \quad\left\{\mathscr{L}_{4}\right\}=[\xi, 0]^{T}, \\
& \left\{\mathscr{L}_{5}\right\}=\left[0,\left(3 \xi^{2}-1\right)\right]^{T}, \quad\left\{\mathscr{L}_{6}\right\}=\left[\left(3 \xi^{2}-1\right), 0\right]^{T}
\end{aligned}
$$

\subsection{Field-inconsistent and field-consistent projections}

Using the two-noded Timoshenko beam as illustration, Mukherjee \& Prathap (2001) have shown that the subspace $\mathfrak{B}$ originating from the strain-displacement operators is fieldinconsistent, and yields locked strain projections with spurious stress oscillations if the subspace cannot be spanned by the corresponding Legendre Orthogonals for the parent space $\mathfrak{P}_{n}^{r}$. In general, locking and spurious stress oscillations result only in multi-component strain vectors when the subspace $\mathfrak{B}$ cannot be spanned by the standard basis vectors of the parent space. Field-inconsistent finite element solutions, as a rule, are variationally correct, for they satisfy the projection theorems (energy-error rule); they agree with the orthogonal projections (best-fits) of the analytical strain vectors onto subspace $\mathfrak{B}$. Elements (like the simple bar element and the Euler beam element) involving single-strain component, do not lock, for their formulations are always field-consistent since strains having single component can always be expressed as a linear combination of Legendre polynomials. Field-consistent formulations involve strain projections in which spurious strain oscillations and locking are absent. In these formulations, the strains are effectively projected on field-consistent subspaces, that can be spanned by standard basis vectors of the parent space.

Locking and the associated spurious stress oscillations in field-inconsistent elements are generally suppressed through reduced integration (an extra-variational method) for deriving the stiffness matrix. As a general rule, a field-consistent finite element solution obtained through reduced integration is variationally correct (i.e. agrees with the best-fits, or orthogonal projections onto an artificially generated field-consistent subspace $\mathfrak{B}^{*}$ ) only if

$$
\int_{\text {ele }}[B]^{T}[D]\{\varepsilon\} \mathrm{d} x=\int_{\text {ele }}\left[B^{*}\right]^{T}[D]\{\varepsilon\} \mathrm{d} x,
$$

where $[B]$ is the original field-inconsistent strain-displacement matrix, and $\left[B^{*}\right]$ is the effective field-consistent strain displacement matrix from the reduced integration process. If (10) is violated, the field-consistent finite element solution obtained through an extra-variational technique like reduced integration will not be variationally correct (i.e., will deviate from the orthogonal projection of the analytical strain vector onto the field-consistent subspace $\mathfrak{B}^{*}$ by an extraneous response). For instance, reduced integration-induced field-consistent finite element results are variationally incorrect with the three-noded beam element having a distributed load that varies with $\xi$ linearly. Further research is being made to establish a rigorous and general method based on the function space approach to test the variational correctness of reduced integration-induced finite element solutions. 
The examples considered in this paper involve loading conditions so that (10) is satisfied. Therefore the field-consistent finite element results for such problems, obtained through reduced integration, are variationally correct. The field-consistent finite element strain vectors can therefore be predicted directly, using (3), as orthogonal projections of the analytical strain vector $\{\varepsilon\}$ on an artificially generated field-consistent subspace $\mathfrak{B}^{*}$ that can be spanned by the standard basis vectors.

\section{Field inconsistent and field consistent solutions of the three-noded Timoshenko beam element}

\subsection{Field-inconsistent solution}

The three-noded isoparametric beam element, formulated to cater to curved geometry in the plane, is shown in figure $2 \mathrm{a}$. The general geometry and displacement field of this element are given by

$$
\begin{array}{rl}
x=\sum_{i=1}^{3} N_{i} x_{i} & y=\sum_{i=1}^{3} N_{i} y_{i} \\
w=\sum_{i=1}^{3} N_{i} w_{i} & \theta=\sum_{i=1}^{3} N_{i} \theta_{i}
\end{array}
$$

where the quadratic Lagrangian shape functions are given by

$$
N_{1}=-\xi(1-\xi) / 2, \quad N_{2}=1-\xi^{2}, \quad \text { and } \quad N_{3}=\xi(1+\xi) / 2 .
$$

(a)

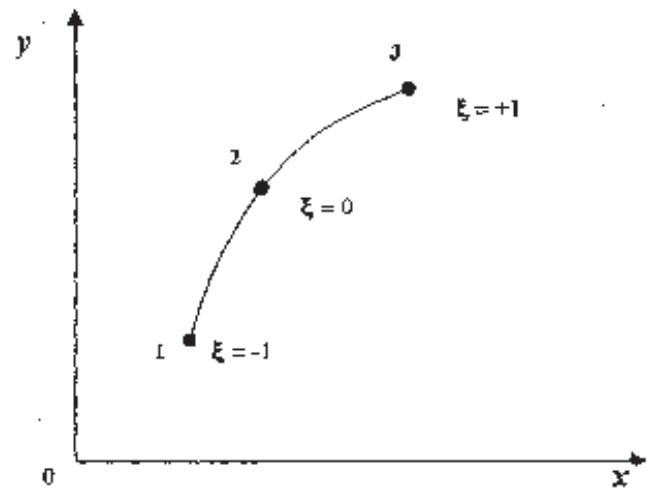

(b) $W_{1}$

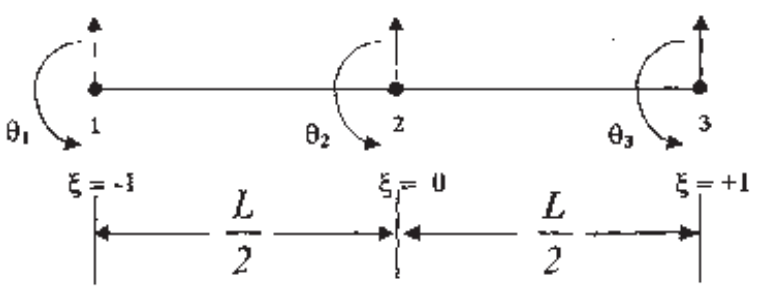

Figure 2. Isoparametric three-noded Timoshenko beam element. (a) General two-dimensional curved beam element, (b) straight beam element. 
We restrict ourselves to the special case of the straight beam, of length $L$ (figure $2 b$ ) to demonstrate in a simple fashion the principle behind the generation of field-inconsistent and field-consistent solutions. For that reason, we may express the geometry simply as a linear function of the non-dimensional coordinate $\xi$, with origin at the beam center, the position of the middle node.

$$
x=\xi(L / 2), \quad y=0 .
$$

The finite element strain vector is given by

$$
\begin{aligned}
(\bar{\varepsilon}) & =\left(\begin{array}{l}
\mathrm{d} \theta / \mathrm{d} x \\
\theta-\mathrm{d} w / \mathrm{d} x
\end{array}\right) \\
& =\left[\begin{array}{cccccc}
0 & \frac{(2 \xi-1)}{L} & 0 & \frac{-4 \xi}{L} & 0 & \frac{(2 \xi+1)}{L} \\
-\frac{(2 \xi-1)}{L} & -\frac{\xi(1-\xi)}{2} & \frac{4 \xi}{L} & \left(1-\xi^{2}\right) & -\frac{(2 \xi+1)}{L} & \frac{\xi(1+\xi)}{2}
\end{array}\right]\left\{\delta^{e}\right\} \\
& =[B]\left\{\delta^{e}\right\},
\end{aligned}
$$

where $\left\{\delta^{e}\right\}$ is the element nodal displacement vector, $\left\{\delta^{e}\right\}=\left[w_{1}, \theta_{1}, w_{2}, \theta_{2}, w_{3}, \theta_{3}\right]^{T}$. The rigidity matrix for the element is given by

$$
[D]=\left[\begin{array}{cc}
E I & 0 \\
0 & k G A
\end{array}\right],
$$

where $E I$ and $k G A$ represent respectively the bending and shear rigidities of the beam section. Since there are six degrees of freedom of the element, the strain-displacement matrix $[B]$ consists of six column vectors. These vectors are not all linearly independent, showing that there are inherent rigid body motions in the element. We thus expect the dimension of the subspace $\mathfrak{B}$, originating from the six column vectors of $[B]$ matrix , to be $m=6-2=4$, assuming two rigid body motions. Using the Gram-Schmidt procedure, we find the four orthogonal basis vectors spanning the four-dimensional subspace $\mathfrak{B},\left(\mathfrak{B} \subset \mathfrak{P}_{3}^{2}\right)$ as

$$
\begin{aligned}
& \left\{u_{1}\right\}=\left\{\begin{array}{l}
0 \\
\xi
\end{array}\right\}, \quad\left\{u_{2}\right\}=\left\{\begin{array}{l}
0 \\
1
\end{array}\right\}, \quad\left\{u_{3}\right\}=\left\{\begin{array}{c}
(2 \xi-1) / L \\
\left(3 \xi^{2}-1\right) / 6
\end{array}\right\}, \quad \text { and } \\
& \left\{u_{4}\right\}=\left\{\begin{array}{c}
\{2 \xi(1-\lambda)+(1+\lambda)\} / L \\
(1-\lambda)\left(3 \xi^{2}-1\right) / 6
\end{array}\right\},
\end{aligned}
$$

where

$$
\lambda=\frac{1+(4 e / 5)}{7+(4 e / 5)}, \quad e=\frac{k G A L^{2}}{12 E I} .
$$

One may normalize the basis vector $\left\{u_{4}\right\}$ further, through division by $(1-\lambda)$, so that a simpler form can be obtained as

$$
\left\{u_{4}\right\}=\left\{\begin{array}{c}
(2 \xi+\kappa) / L \\
\left(3 \xi^{2}-1\right) / 6
\end{array}\right\}, \quad \text { where } \quad \kappa=\frac{1+\lambda}{1-\lambda}=\frac{4(e+5)}{15} .
$$


It should be noted that for this beam element, the inner product of two vectors $\{a\}$ and $\{b\}$, each of two rows, $(r=2)$, is defined as

$$
\langle a, b\rangle=\int_{-1}^{1}\{a\}^{T}[D]\{b\} \frac{L}{2} \mathrm{~d} \xi .
$$

Since not all the orthogonal basis vectors spanning the subspace $\mathfrak{B}$ are Legendre Orthogonals, we can infer that this subspace is field-inconsistent (Mukherjee \& Prathap 2001). The finite element strain vector, as projection of the analytical strain vector onto this field-inconsistent subspace $\mathfrak{B}$, is given by

$$
\{\bar{\varepsilon}\}=\sum_{i=1}^{4} \frac{\left\langle u_{i}, \varepsilon\right\rangle}{\left\langle u_{i}, u_{i}\right\rangle}\left\{u_{i}\right\}
$$

\subsection{Field-consistent solution}

Conventionally, field-consistent finite element solutions in elements are obtained through reduced integration of the stiffness matrix. Exact integration for the element stiffness matrix of the three-noded beam element, given by

$$
\left[K^{e}\right]=\int_{-L / 2}^{L / 2}[B]^{T}[D][B] \mathrm{d} x=\frac{L}{2} \int_{-1}^{1}[B]^{T}[D][B] \mathrm{d} \xi,
$$

requires a three-point rule by Gaussian quadrature, leading to field-inconsistent solution. For field-consistent finite element solution, the two-point rule is adopted, effectively yielding an element field-consistent stiffness matrix $\left[K^{e *}\right]$, given as

$$
\left[K^{e *}\right]=\int_{-L / 2}^{L / 2}\left[B^{*}\right]^{T}[D]\left[B^{*}\right] \mathrm{d} x=\frac{L}{2} \int_{-1}^{1}\left[B^{*}\right]^{T}[D]\left[B^{*}\right] \mathrm{d} \xi,
$$

where $\left[B^{*}\right]$ is the field-consistent strain-displacement vector, given by the following expression of the field-consistent strain vector,

$$
\begin{aligned}
\left\{\bar{\varepsilon}^{*}\right\} & =\left[\begin{array}{cccccc}
0 & \frac{(2 \xi-1)}{L} & 0 & \frac{-4 \xi}{L} & 0 & \frac{(2 \xi+1)}{L} \\
-\frac{(2 \xi-1)}{L} & -\frac{\{\xi-(1 / 3)\}}{2} & \frac{4 \xi}{L} & \frac{2}{3} & -\frac{(2 \xi+1)}{L} & \frac{\{\xi+(1 / 3)\}}{2}
\end{array}\right]\left\{\delta^{e}\right\} \\
& =\left[B^{*}\right]\left\{\delta^{e}\right\} .
\end{aligned}
$$

The field-consistent matrix $\left[B^{*}\right]$ is obtained from the field-inconsistent $[B]$ of (14), by first expressing $\xi^{2}$ in terms of the Legendre quadratic polynomial as

$$
\xi^{2}=\left(3 \xi^{2}-1\right)+1 / 3=P_{3}+1 / 3,
$$

and then dropping the Legendre polynomial $P_{3}=\left(3 \xi^{2}-1\right)$. Thus by replacing $\xi^{2}$ of the field-inconsistent $[B]$ by $(1 / 3)$, the field-consistent $\left[B^{*}\right]$ is obtained.

Using the Gram-Schmidt procedure, the orthogonal basis vectors spanning the $\mathfrak{B}^{*}$ subspace originating from the column vectors of the field-consistent strain displacement matrix $\left[B^{*}\right]$, can be obtained as

$$
\left\{u_{1}^{*}\right\}=\left\{\begin{array}{c}
1 \\
0
\end{array}\right\}, \quad\left\{u_{2}^{*}\right\}=\left\{\begin{array}{c}
0 \\
1
\end{array}\right\}, \quad\left\{u_{3}^{*}\right\}=\left\{\begin{array}{c}
\xi \\
0
\end{array}\right\} \quad \text { and } \quad\left\{u_{4}^{*}\right\}=\left\{\begin{array}{l}
0 \\
\xi
\end{array}\right\} .
$$


Since this four-dimensional subspace $\mathfrak{B}^{*}\left(\mathfrak{B}^{*}=\mathfrak{P}_{2}^{2}\right)$ can be spanned by the Legendre Orthogonals, that are the standard basis vectors, it is field-consistent for strain projections. This subspace is artificially generated through the reduced integration process. The field-consistent best-fit strain vector is obtained as the orthogonal projection of the analytical strain vector onto this field-consistent subspace $\mathfrak{B}^{*}$ from the expression

$$
\left\{\bar{\varepsilon}^{*}\right\}=\sum_{i=1}^{4} \frac{\left\langle u_{i}^{*}, \varepsilon\right\rangle}{\left\langle u_{i}^{*}, u_{i}^{*}\right\rangle}\left\{u_{i}^{*}\right\} .
$$

This equation can be used to make a priori estimates of the field-consistent finite-element solutions determined from reduced integration process, provided the following condition, that guarantees variational correctness of such solutions, is satisfied

$$
\frac{L}{2} \int_{-1}^{1}[B]^{T}[D]\{\varepsilon\} \mathrm{d} \xi=\frac{L}{2} \int_{-1}^{1}\left[B^{*}\right]^{T}[D]\{\varepsilon\} \mathrm{d} \xi .
$$

If (23) is violated, finite element solutions with reduced integration will not be variationally correct, and will deviate from the orthogonal projections onto the artificially generated subspace $\mathfrak{B}^{*}$ (or best-fits) given by (22) by an extraneous response of the field-consistent element to a self-equilibrating, spurious load vector, given by

$$
\left\{F_{E}^{e}\right\}=-\frac{L}{2} \int_{-1}^{1}\left[[B]-\left[B^{*}\right]\right]^{T}[D]\{\varepsilon\} \mathrm{d} \xi .
$$

Further research is in progress to establish a general method to predict the variational correctness of reduced integration induced finite element solutions. In this paper, nodal loads and uniform distributed loads are considered so that (23) is satisfied. Therefore reduced integration induced field-consistent solutions are variationally correct and can be predicted directly from the best-fit strain expression of (22).

\section{Some solutions using the three noded element and error estimation}

\subsection{Conventional finite element analysis}

As illustration, the cantilever beam with different loading conditions is analysed using a single element, with field-inconsistent and consistent formulations. From (11) the conventional compact form of the displacement field is given by

$$
\left\{\begin{array}{c}
w \\
\theta
\end{array}\right\}=[N]\left\{\delta^{e}\right\}
$$

where $[N]$ is the quadratic Lagrangian shape function matrix of size $2 \times 6$. Using the conventional methods of finite element analysis, one first solves for the unknown nodal displacement $\left\{\delta^{e}\right\}$ for the element from the equation,

$$
\left[K^{e}\right]\left\{\delta^{e}\right\}=\left\{F^{e}\right\}+\left\{R^{e}\right\},
$$

where the element stiffness matrix $\left[K^{e}\right]$ and the nodal applied generalised force vector $\left\{F^{e}\right\}$ are respectively given by

$$
\left[K^{e}\right]=\int_{-1}^{1}[B]^{T}[D][B] \frac{L}{2} \mathrm{~d} \xi, \quad\left\{F^{e}\right\}=\int_{-1}^{1}[N]^{T}\{\rho\} \frac{L}{2} \mathrm{~d} \xi .
$$


Here $\{\rho\}=[\rho, \mu]^{T}$ represents the distributed load intensity (transverse load $\rho$ and moment $\mu$ per unit beam length). In general, when multiple elements are taken in the analysis, the vector $\left\{R^{e}\right\}$ for a particular element represents the reaction vector acting on the element from adjacent elements/supports through nodal connections. For a single element, the force vector $\left\{R^{e}\right\}$ is the reaction vector from boundary constraints at nodes. For our problem of the cantilever beam discretised using a single element, the nodal point 3 (where $\xi=+1$ ) is clamped, so the boundary conditions are $w_{3}=\theta_{3}=0$ (figures 3 and 4). The initially unknown vector $\left\{R^{e}\right\}$ corresponding to these nodal boundary kinematic conditions does not appear in the following reduced equation where rigid body motions are eliminated,

(a)
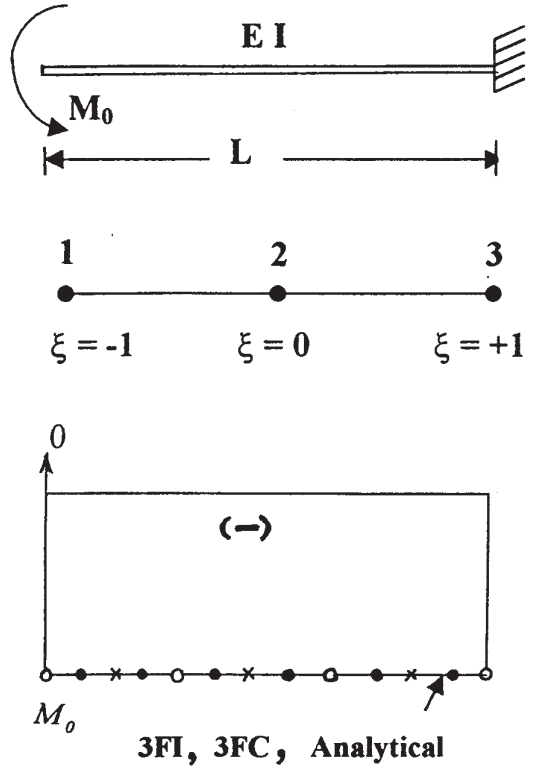

Bending moment

Zero shear force
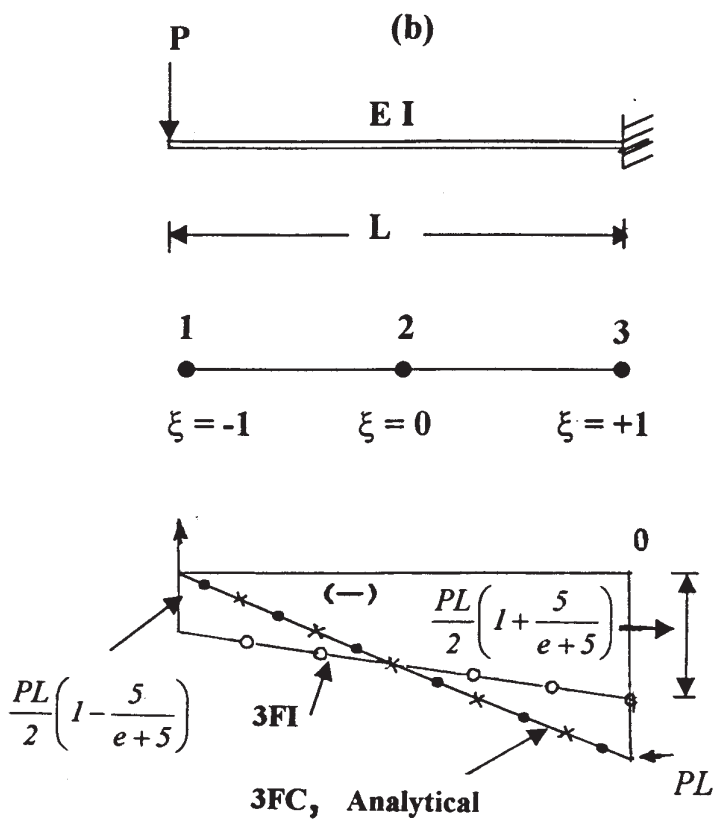

Bending moment

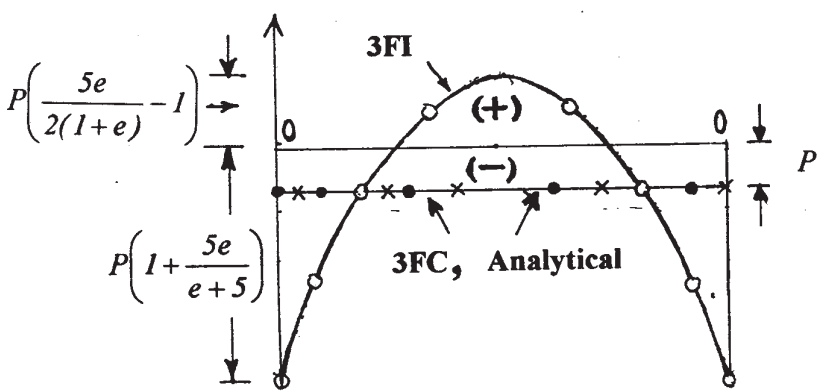

Shear force

Figure 3. Analysis of cantilever beam using a single three-noded beam element under different nodal loads. Here $e=k G A L^{2} /(12 E I)$. 3FI-field inconsistent solution; 3FC-field consistent solution. (a) Tip moment, (b) tip transverse load. 

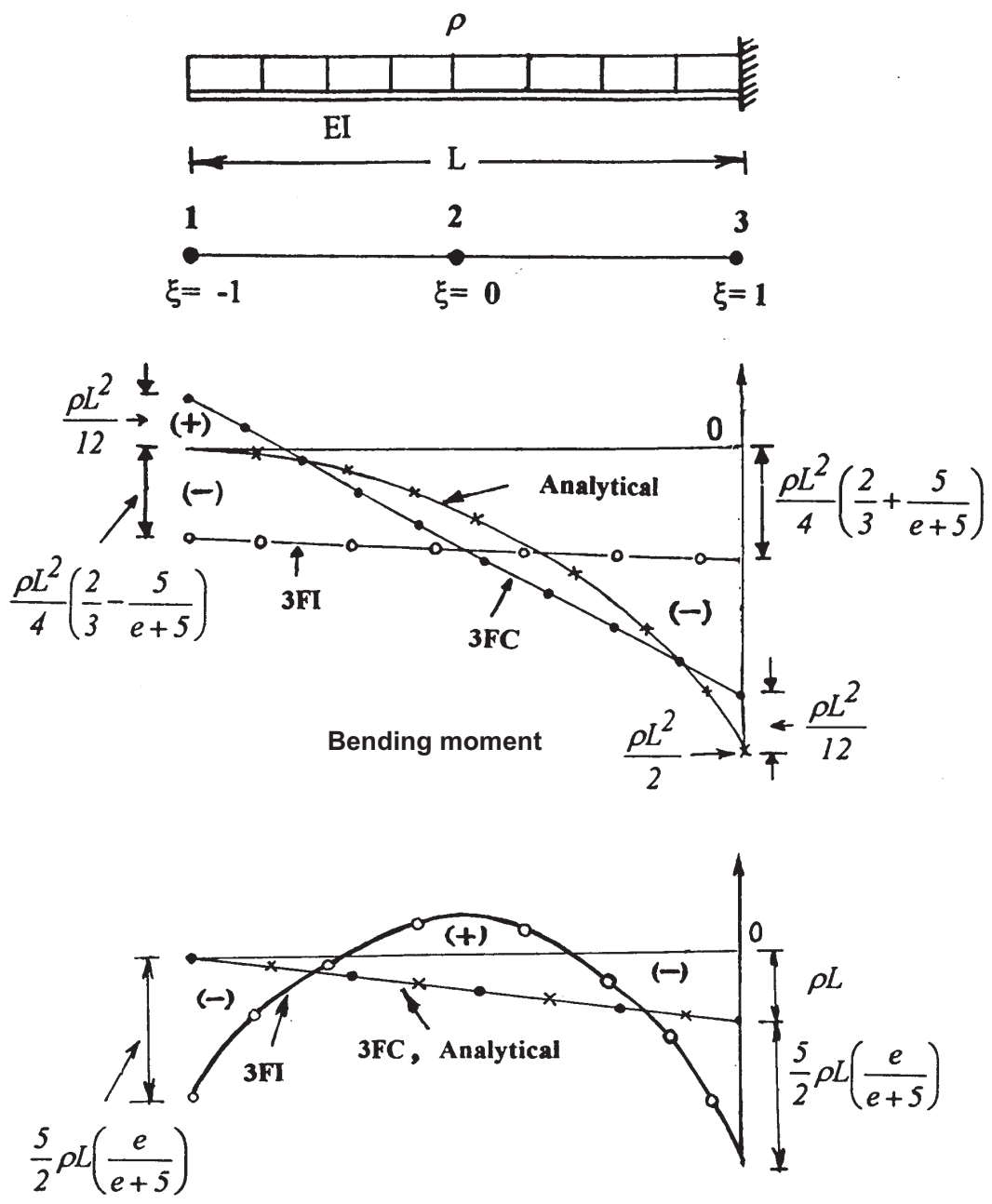

Shear force

Figure 4. Analysis of cantilever beam using a single three-noded beam element subjected to a uniformly distributed load of intensity $\rho$. Here $e=k G A L^{2} /(12 E I)$. 3FI- field inconsistent solution; 3FC- field consistent solution.

$$
[K]\left\{\delta^{e}\right\}=\{F\} .
$$

In practice, (28) is actually solved for determining the unknown displacement vector $\left\{\delta^{e}\right\}$. Here the modified stiffness matrix $[K]$ and the force vector $\{F\}$ are derived respectively from $\left[K^{e}\right]$ and $\left\{F^{e}\right\}$ using the conventional penalty methods to incorporate the boundary conditions. After solving for the unknown nodal displacement vector $\left\{\delta^{e}\right\}$, the element strain vector $\{\bar{\varepsilon}\}$, the element stress resultant vector $\{\bar{\sigma}\}$ and the reaction vector $\left\{R^{e}\right\}$ can be obtained from the following expressions

$$
\begin{aligned}
\{\bar{\varepsilon}\} & =[B]\left\{\delta^{e}\right\}, \quad\{\bar{\sigma}\}=[D][B]\left\{\delta^{e}\right\}, \\
\left\{R^{e}\right\} & =\left[K^{e}\right]\left\{\delta^{e}\right\}-\left\{F^{e}\right\} .
\end{aligned}
$$


A field-inconsistent element stiffness matrix $\left[K^{e}\right]$ will involve a field-inconsistent straindisplacement matrix $[B]$. To avoid field-inconsistency for the quadratic beam element, a reduced integration scheme is adopted, i.e., a two-point rule for Gauss integration for stiffness matrix is used instead of the necessary three-point rule. This step effectively replaces the field-inconsistent $[B]$ matrix by the field-consistent $\left[B^{*}\right]$ matrix. The results obtained from the finite element codes based on the conventional formulation and procedures agree with those predicted by the function space approach.

\subsection{The function space projection method}

The function space projection approach is used here to derive element strain vectors and $a$ priori error estimates for the cantilever beam with a single element discretization (figures 3 and 4$)$. The entire beam is taken as a single element, clamped at the nodal point $3(\xi=+1)$. The results of the analyses are presented in tables 1 and 2 .

4.2a Cantilever beam subjected to a tip moment $M_{0}$ (figure $3 a$ ): This is a case of a beam subjected to pure moment $M_{0}$, applied at the tip (node 1 where $\xi=-1$ ). The finite element strain vector in the field inconsistent solution is as good as the field consistent solution, for both are identical to the analytical strain vector.

$$
\{\varepsilon\}=\{\bar{\varepsilon}\}=\left\{\bar{\varepsilon}^{*}\right\}=\left\{\begin{array}{c}
-M_{0} / E I \\
0
\end{array}\right\} .
$$

Table 1. Field-inconsistent (3FI) and field-consistent (3FC) solutions of the cantilever beam, subjected to nodal loads, using a single element.

\begin{tabular}{|c|c|c|}
\hline & $\begin{array}{l}\text { Cantilever with tip } \\
\text { moment } M_{0} \text { (figure 3a) }\end{array}$ & $\begin{array}{c}\text { Cantilever with tip load } P \\
\text { (figure } 3 \mathrm{~b} \text { ) }\end{array}$ \\
\hline $\begin{array}{l}\text { Analytical strain vector } \\
\text { (hogging bending strain } \\
\text { and anticlockwise shear } \\
\text { couple are negative) }\end{array}$ & $\{\varepsilon\}=\left\{\begin{array}{c}-M_{0} / E I \\
0\end{array}\right\}$ & $\{\varepsilon\}=\left\{\begin{array}{c}-P L(1+\xi) /(2 E I) \\
-P / k G A\end{array}\right\}$ \\
\hline $\begin{array}{l}\text { Field inconsistent }(3 \mathrm{FI}) \\
\text { strain vector and error } \\
\text { norm squared value }\end{array}$ & $\begin{array}{l}\{\bar{\varepsilon}\}=\left\{\begin{array}{c}-M_{0} / E I \\
0\end{array}\right\} \\
\|q\|^{2}=0\end{array}$ & $\begin{array}{l}\{\bar{\varepsilon}\}=\left\{\begin{array}{l}-\frac{P L}{2 E I}\left(1+\frac{5}{e+5} \xi\right) \\
-\left[\frac{P}{k G A}+\frac{P L^{2}}{2 E I} \frac{5\left(3 \xi^{2}-1\right)}{12(e+5)}\right]\end{array}\right. \\
\|q\|^{2}=\frac{L}{2} \frac{(P L)^{2}}{6 E I}\left(\frac{e}{e+5}\right)\end{array}$ \\
\hline $\begin{array}{l}\text { Field consistent ( } 3 \mathrm{FC}) \\
\text { strain vector and error } \\
\text { norm squared value }\end{array}$ & $\begin{array}{l}\left\{\bar{\varepsilon}^{*}\right\}=\left\{\begin{array}{c}-M_{o} / E I \\
0\end{array}\right\} \\
\left\|q^{*}\right\|^{2}=0\end{array}$ & $\begin{array}{l}\left\{\bar{\varepsilon}^{*}\right\}=\left\{\begin{array}{c}-P L(1+\xi) /(2 E I) \\
-P / k G A\end{array}\right\} \\
\left\|q^{*}\right\|^{2}=0\end{array}$ \\
\hline
\end{tabular}

$$
e=\frac{k G A L^{2}}{12 E I}, \quad\|q\|^{2}=(L / 2) \int_{-1}^{1}\{q\}^{T}[D]\{q\} \mathrm{d} \xi
$$


Table 2. Field-inconsistent (3FI) and field-consistent (3FC) solutions of the cantilever beam subjected to uniformly distributed load (figure 4), using a single element..

Analytical strain vector (hogging bending strain and anticlockwise shear couple are negative).

Field-inconsistent (3FI) strain vector

Field-consistent

$(3 \mathrm{FC})$ strain vector

$$
\{\varepsilon\}=\left\{\begin{array}{c}
-\frac{\rho L^{2}}{8 E I}(1+\xi)^{2} \\
-\frac{\rho L}{2 k G A}(1+\xi)
\end{array}\right\}
$$

$$
\{\bar{\varepsilon}\}=\left\{\begin{array}{c}
-\frac{\rho L^{2}}{4 E I}\left(\frac{5}{e+5} \xi+\frac{2}{3}\right) \\
-\left[\frac{\rho L}{2 k G A}(1+\xi)+\frac{\rho L^{3}}{8 E I}\left(\frac{5}{e+5}\right)\left(\frac{3 \xi^{2}-1}{6}\right)\right]
\end{array}\right\}
$$

$$
\left\{\bar{\varepsilon}^{*}\right\}=\left\{\begin{array}{c}
-\frac{\rho L^{2}}{4 E I}\left(\xi+\frac{2}{3}\right) \\
-\frac{\rho L}{2 k G A}(1+\xi)
\end{array}\right\}
$$

Here the load per unit length is denoted by $\rho$, and $e=k G A L^{2} /(12 E I)$.

The stress resultant vectors are thus given by

$$
\{\bar{\sigma}\}=\left\{\bar{\sigma}^{*}\right\}=\left\{\begin{array}{c}
-M_{0} \\
0
\end{array}\right\} .
$$

Thus for the case of pure moment, both the field-inconsistent (3FI) and field-consistent (3FC) solutions yield lock-free results, with no shear oscillations (table 1). This is a case of pure coincidence, and it should be noted that the element is not purely lock-free for arbitrary loading, for the subspace $\mathfrak{B}$ is field-inconsistent. Such an observation has been earlier made by Prathap (1993), and an explanation for this phenomenon is presented using the fieldconsistency paradigm.

4.2b Cantilever beam subjected to a tip transverse load P (figure $3 b$ ): The results of the analyses are presented explicitly in Table 1 . The quadratic shear strain oscillations in the fieldinconsistent (3FI) solution can be noticed. The parameter $e=k G A L^{2} /(12 E I)$, reflecting the non-dimensional rigidity ratio, plays an important part in the field-inconsistent formulation. The error norm squared value for the analysis using a single element is given by

$$
\|q\|^{2}=\frac{(P L)^{2} L}{12 E I}\left(\frac{e}{e+5}\right) .
$$

The norm squared values of the analytical strain vector and the field-inconsistent strain vector are given by the following expressions

$$
\begin{aligned}
& \|\varepsilon\|^{2}=\langle\varepsilon, \varepsilon\rangle=\frac{(P L)^{2} L}{12 E I}\left(4+\frac{1}{e}\right), \\
& \|\bar{\varepsilon}\|^{2}=\langle\bar{\varepsilon}, \bar{\varepsilon}\rangle=\frac{(P L)^{2} L}{12 E I}\left(\frac{5}{e+5}+\frac{1}{e}+3\right) .
\end{aligned}
$$


From $(31 \mathrm{a}-\mathrm{c})$ it can be easily shown that even the field-inconsistent solution satisfies the projection theorems given by (6a) and (6b), i.e. it is variationally correct. The field-inconsistent element stress resultant vector is given as

$$
\{\bar{\sigma}\}=\left\{\begin{array}{c}
\bar{M} \\
\bar{V}
\end{array}\right\}=[D]\{\bar{\varepsilon}\}=\left\{\begin{array}{c}
-\left(\frac{P L}{2}+\frac{P L}{2} \frac{5}{e+5} \xi\right) \\
-\left[P+\frac{5}{2} P \frac{e}{e+5}\left(3 \xi^{2}-1\right)\right]
\end{array}\right\} .
$$

As the beam becomes thinner, the parameter $e=k G A L^{2} /(12 E I)$ increases. Thus the limiting case of the field-inconsistent formulation for very thin beams is represented by the following limits,

$$
\operatorname{Lim}_{e \rightarrow \infty}\{\bar{\sigma}\}=\left\{\begin{array}{c}
-\frac{P L}{2} \\
-\left[P+\frac{5}{2} P\left(3 \xi^{2}-1\right)\right]
\end{array}\right\}, \quad \operatorname{Lim}_{e \rightarrow \infty}\|q\|^{2}=\frac{(P L)^{2} L}{12 E I}
$$

These expressions are in complete agreement with numerical results from finite element computer codes. It is obvious that the oscillations in the shear strain and shear stress resultant are quadratic in nature. Interestingly, it may be observed from table 1 and (33a) that as the beam gets thinner (increasing $e$ ), the linear parts of the bending strain and bending moment gradually die out, and these tend to flatten out to constant non-zero values. Furthermore, while the quadratic oscillations for the shear strain component die out with increasing values of $e$, resulting from increasing slenderness of the beam, the quadratic oscillations for the shear stress resultant persist. It is evident that the quadratic oscillatory part of the shear strain for the field inconsistent ( $3 F I)$ formulation behaves like the Euler beam formulation in the limiting case of the thin beam, where shear strains vainish, but finite values of shear stress resultant persist, due to the large ratio of the shear rigidity to the bending rigidity.

As expected, there are no locking and shear oscillations in the field-consistent (3FC) solution. For nodal loading, the solution is identical to the analytical strain vector with linear variation of the bending strain with the coordinate $\xi$ (table 1, figure $3 \mathrm{~b}$ ). The field-consistent stress resultant vector can also be obtained by substituting $e=0$ in the corresponding expressions for the field-inconsistent stress resultant vector.

4.2c Cantilever beam subjected to uniformly distributed load (figure 4): The uniform load intensity (transverse load per unit length) is represented here by $\rho$. The results of the analysis are presented in table 2 . The field-inconsistent element stress resultant vector is given by

$$
\{\bar{\sigma}\}=\left\{\begin{array}{c}
\bar{M} \\
\bar{V}
\end{array}\right\}=[D]\{\bar{\varepsilon}\}=\left\{\begin{array}{c}
-\frac{\rho L^{2}}{4}\left(\frac{5}{e+5} \xi+\frac{2}{3}\right) \\
-\left[\frac{\rho L}{2}(1+\xi)+\frac{5}{4} \rho L\left(\frac{e}{e+5}\right)\left(3 \xi^{2}-1\right)\right]
\end{array}\right\} .
$$


It can be observed that for the distributed load case the field-inconsistent solution shows behaviour similar to that of the nodal point load case. With decreasing thickness (or increasing $e$ value) the quadratic oscillatory part of the shear strain tends to vanish, the shear force oscillation tends towards a saturation quadratic function independent of $e$, and both the bending strain (curvature) and bending moment tend to flatten out to constants.

$$
\operatorname{Lim}_{e \rightarrow \infty}\{\bar{\sigma}\}=\left\{\begin{array}{c}
-\frac{\rho L^{2}}{6} \\
-\left[\frac{\rho L}{2}(1+\xi)+\frac{5}{4} \rho L\left(3 \xi^{2}-1\right)\right]
\end{array}\right\} .
$$

Again it may be noted that the field-consistent stress resultant vector can also be obtained by substituting $e=0$ in the corresponding expressions for the field-inconsistent stress resultant vector. As expected, there are no locking and shear oscillations in the field-consistent (3FC) solution (figure 4).

\section{Comparison of the three-noded beam element with the two-noded beam element}

We first review briefly the two-noded Timoshenko beam element for the purpose of comparison with the three noded Timoshenko beam element.

\subsection{Field-inconsistent and field-consistent formulations of the two-noded beam element}

Mukherjee \& Prathap (2001) have presented an explanation for shear locking in the fieldinconsistent isoparametric two-noded Timoshenko beam element, in which linear Lagrangian shape functions are used for interpolation of the geometry and displacement field.

Orthogonal basis vectors spanning the original field-inconsistent, two-dimensional $(m=2)$ subspace $\mathfrak{B}$ are given as

$$
\left\{u_{1}\right\}=\left\{\begin{array}{l}
0 \\
1
\end{array}\right\} \quad \text { and } \quad\left\{u_{2}\right\}=\left\{\begin{array}{c}
2 / L \\
\xi
\end{array}\right\} .
$$

The basis vector $\left\{u_{2}\right\}$ is not a Legendre Orthogonal, and therefore contributes to the field-inconsistency problem. Orthogonal basis vectors spanning the field-consistent, twodimensional $(m=2)$ subspace $\mathfrak{B}^{*}$ (artificially generated through reduced integration) are given as

$$
\left\{u_{1}^{*}\right\}=\left\{\begin{array}{c}
0 \\
1
\end{array}\right\} \quad \text { and } \quad\left\{u_{2}^{*}\right\}=\left\{\begin{array}{l}
1 \\
0
\end{array}\right\},
$$

which are Legendre Orthogonals. It has been shown how by adopting the technique of reduced integration, i.e. using the one-point Gaussian quadrature rule (instead of the necessary twopoint rule for exact integration for the stiffness matrix involving originally field-inconsistent strains) the field-consistent finite element solution is effectively obtained. Using (3), with $m=2$ and the appropriate basis vectors, the field-inconsistent finite element strain vectors can be obtained as orthogonal projections of the analytical strain vector onto the $\mathfrak{B}$ subspace. For beams with only nodal loading, (10) is satisfied, making the reduced integration induced field-consistent finite element results match the field-consistent best-fits obtained from (3) with field-consistent (standard) orthogonal basis vectors. However, reduced integrationinduced field-consistent finite element results for the two-noded element are, in general, variationally incorrect with distributed loading conditions. 
(a)

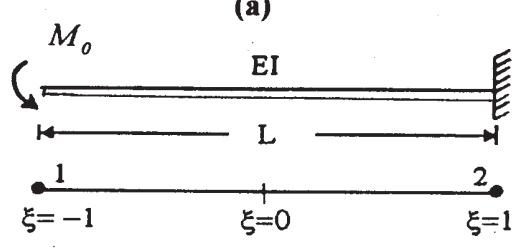

(b)

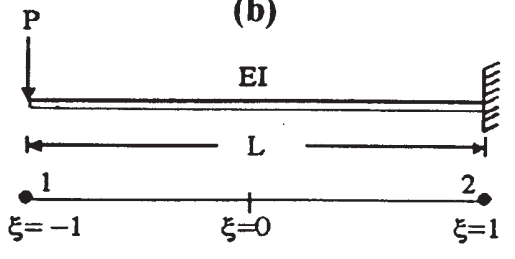

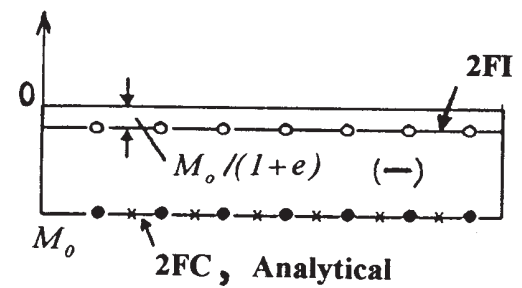

Bending moment

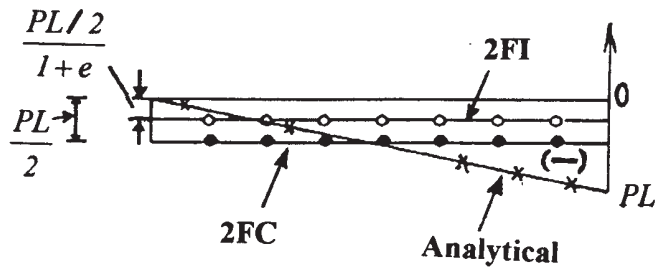

Bending moment

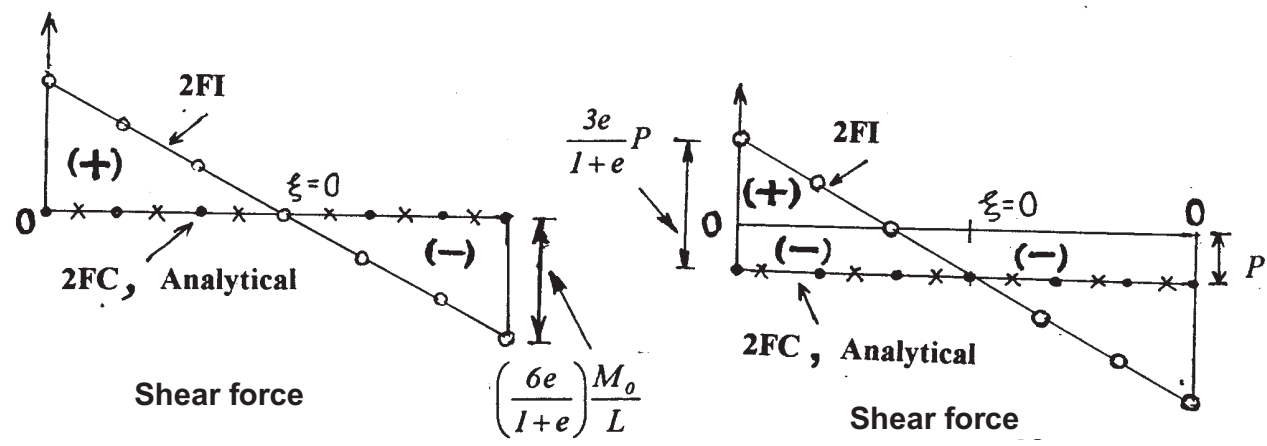

Figure 5. Cantilever beam analysis using a single two-noded beam element under different nodal loads. 2FI- field inconsistent (locked) solution; 2FC- field consistent (lockfree) solution. (a) Tip moment (b) tip transverse load.

In thin beams, severe locking and linear shear oscillations occur in the field-inconsistent solution of the two noded element $(2 \mathrm{FI})$, while such features are completely eliminated in the field-consistent solution (2FC). The results of analysis of the cantilever beam with a single two-noded Timoshenko beam element (figures 5a and b), and the corresponding error norm squared values are presented explicitly in table 3 . Both solutions satisfy the projection theorem, viz., (6a) and (6b).

5.2 Comparisons between the field-inconsistent solutions of the three-noded element (3FI) with two-noded element solutions (2FI and $2 F C$ )

We show here how the behaviour of the three-noded element is remarkably different from that of the two-noded element. Results of the analysis using the two- and three-noded elements for the limiting case of thin beam are presented in table 4. 


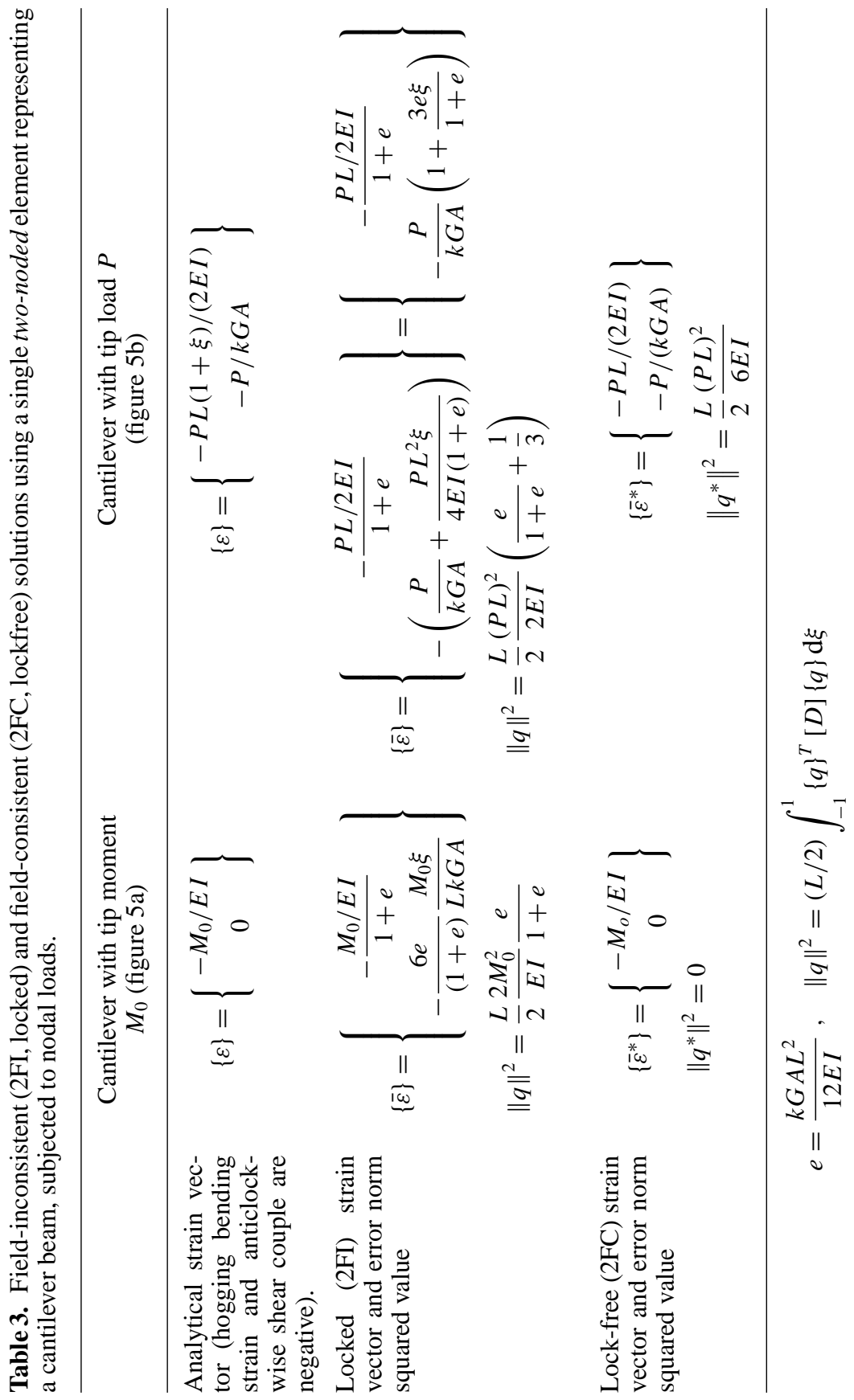


Table 4. Comparison of results from the field-inconsistent three-noded element (3FI) with those of the field-inconsistent (2FI) and field-consistent (2FC) two-noded elements for the limiting case of a very thin beam ( $e$ is very large).

\begin{tabular}{|c|c|c|}
\hline & $\begin{array}{l}\text { Cantilever with tip } \\
\text { moment } M_{0}\end{array}$ & $\begin{array}{l}\text { Cantilever with tip transverse } \\
\text { load } P\end{array}$ \\
\hline $\begin{array}{l}\text { Field-inconsistent three- } \\
\text { noded element (3FI) }\end{array}$ & $\begin{array}{l}\|q\|^{2}=0 \\
\{\bar{\sigma}\}=\left\{\begin{array}{c}-M_{0} \\
0\end{array}\right\}\end{array}$ & $\begin{array}{l}\|q\|^{2}=\frac{L}{2} \frac{(P L)^{2}}{6 E I} \\
\{\bar{\sigma}\}=\left\{\begin{array}{c}-P L / 2 \\
-\left[P+\frac{5}{2} P .\left(3 \xi^{2}-1\right)\right]\end{array}\right.\end{array}$ \\
\hline $\begin{array}{l}\text { Field-inconsistent } \\
\text { noded element }(2 \mathrm{FI})\end{array}$ & $\begin{array}{l}\|q\|^{2}=\frac{L}{2} \frac{2 M_{0}^{2}}{E I} \\
\{\bar{\sigma}\}=\left\{\begin{array}{c}0 \\
-\frac{6 M_{0} \xi}{L}\end{array}\right.\end{array}$ & $\begin{array}{l}\|q\|^{2}=\frac{L}{2}\left(\frac{2(P L)^{2}}{3 E I}\right) \\
\{\bar{\sigma}\}=\left\{\begin{array}{c}0 \\
-P(1+3 \xi)\end{array}\right.\end{array}$ \\
\hline $\begin{array}{l}\text { Field-consistent two- } \\
\text { noded element }(2 \mathrm{FC})\end{array}$ & $\begin{array}{l}\left\|q^{*}\right\|^{2}=0 \\
\left\{\bar{\sigma}^{*}\right\}=\left\{\begin{array}{c}-M_{0} \\
0\end{array}\right.\end{array}$ & $\begin{array}{l}\left\|q^{*}\right\|^{2}=\frac{L}{2} \frac{(P L)^{2}}{6 E I} \\
\left\{\bar{\sigma}^{*}\right\}=\left\{\begin{array}{c}-P L / 2 \\
-P\end{array}\right\}\end{array}$ \\
\hline
\end{tabular}

Results for the pure bending case of the cantilever reveal that locking and shear oscillations get more intense with increasing $e$ values in the field-inconsistent two-noded element (2FI), tending to reach asymptotically the limiting values presented in table 4 . Analysis with the three-noded field-inconsistent formulation (3FI) shows that the there is no locking at all for pure bending .

For the case of cantilever with nodal transverse load, it is obvious from table 4 that for very thin beams $(e \gg 1)$, the behaviour of the field-inconsistent solutions of the three-noded beam element (3FI) tends towards that of the field-consistent solution of the two-noded beam element (2FC). With decreasing thickness of the 3FI element, the bending strain tends to flatten out to a non-zero constant, while quadratic shear strain oscillations die out. Thus, in the limiting case of the thin beam, the strain components, error norm and convergence rate of the $3 \mathrm{FI}$ element tend towards those of the $2 \mathrm{FC}$ element.

$$
\operatorname{Lim}_{e \rightarrow \infty}\|q\|_{(3 F I)}^{2}=\frac{L}{2} \frac{(P L)^{2}}{6 E I}=\left\|q^{*}\right\|_{(2 F C)}^{2} .
$$

For the pure moment case, of course, we have for both thick and thin beams

$$
\|q\|_{(3 F I)}^{2}=\left\|q^{*}\right\|_{(2 F C)}^{2}=0 .
$$

The convergence rates of the 3FI, 3FC and 2FC formulations for the analysis of the thin cantilever beam with tip transverse load, studied earlier by Prathap (1993), is presented graphically in figure 6 . 


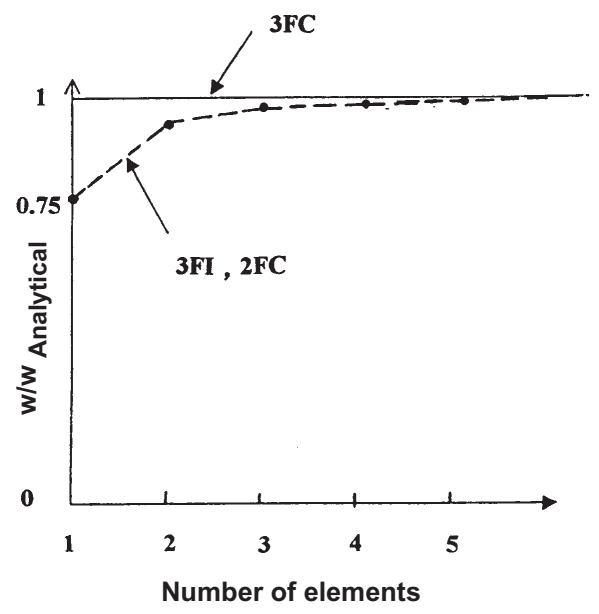

Figure 6. Comparison of the convergence trends of the different formulations of the three-noded element (3FI and 3FC) with the two-noded field-consistent element (2FC) for a very thin beam under nodal transverse load.

\section{Conclusions}

A method based on the function space approach is employed to identify field-consistent and field-inconsistent spaces for strain projections of the three-noded Timoshenko beam element. Numerical results from an in-house finite element computer code confirm that field-inconsistent finite element solutions always agree with the strain projections onto fieldinconsistent subspaces, and are therefore variationally correct. For the loading cases considered here, reduced integration-induced field-consistent finite element solutions agree with the strain projections onto artificially generated field-consistent subspaces, and are therefore variationally correct. This is a fortuitous condition that arises from the vanishing of the spurious force vector, leading to the satisfaction of the normal equations. It has been pointed out that under certain loading conditions, reduced integration induced finite element solutions deviate from the field-consistent best-fits, or orthogonal strain projections by extraneous responses excited by self-equilibrating spurious forces that can be predicted using the function space method.

It has been demonstrated here that contrary to the general faith, the field- inconsistent solutions of the three-noded Timoshenko beam element do not lock severely. The deterioration is of a mild kind, i.e. delayed convergence is the consequence. This behaviour is in sharp contrast with that of the lower order two noded Timoshenko beam element, which locks severely and shows spurious linear shear oscillations in the field-inconsistent formulation, and the rate of convergence of the locked solution is too slow to reach an acceptable level of convergence economically. Furthermore, it has been shown how the field-inconsistent threenoded formulation (3FI) behaves like the field-consistent two-noded (2FC) formulation for the limiting case of very thin beams.

For uniform elements (constant section properties) and rectilinear geometry (constant Jacobian over the element), the standard basis vectors are the Legendre Orthogonals, which are mutually orthogonal with any constant as the kernel function included in the integrand defining the inner product. For non-uniform elements with curved geometry, the characteristic standard basis vectors associated with the corresponding polynomial function space need not be equal to the Legendre Orthogonals, for the associated kernel functions are not necessarily constant over the element, but become functions of the coordinate $\xi$. In practice, determination of the basis vectors for such cases can be tedious, and is beyond the scope 
of the present paper. However, the principle behind identifying field-inconsistent and fieldconsistent solutions remains the same. From the observations made in this paper, one may investigate the possibilities of formulating lock-free, field-consistent elements by first assuming suitable strain-displacement expressions that generate the corresponding field-consistent spaces which can be spanned by the standard basis vectors.

\section{Appendix A - The Gram-Schmidt procedure}

The orthogonal basis vectors $\left\{u_{i}\right\},(i=1,2 . ., m)$, spanning the subspace $\mathfrak{B}$ can be determined from the $N$ column vectors $\left\{b_{j}\right\}(j=1,2, \ldots, N ; N=$ total number of element degrees of freedom) of the matrix $[B]$. The initial basis vector can be taken as any of the column vectors of $[B]$,

$$
\left\{u_{1}\right\}=\left\{b_{1}\right\} .
$$

The other $(m-1)$ non-zero orthogonal basis vectors can be obtained from the general formula

$$
\left\{u_{k+1}\right\}=\left\{b_{k+1}\right\}-\sum_{j=1}^{k} \frac{\left\langle u_{j}, b_{k+1}\right\rangle}{\left\langle u_{j}, u_{j}\right\rangle}\left\{u_{j}\right\}
$$

These basis vectors can be arbitrarily scaled.

\section{List of symbols}

$A \quad$ area of section normal to beam axis;

$\left\{b_{j}\right\} \quad$ column vectors of the $[B]$ matrix;

$[B] \quad$ strain-displacement matrix;

$\mathfrak{B}$ function subspace for strain projections;

$[D] \quad$ rigidity matrix;

$e \quad$ non-dimensional rigidity ratio;

$E \quad$ Young's modulus of beam material;

$\{F\},\left\{F^{e}\right\}$ force vectors;

$G \quad$ shear modulus of beam material;

I section moment of inertia about neutral axis;

$k \quad$ shear correction factor (0.833 for rectangular section);

$[K],\left[K^{e}\right] \quad$ stiffness matrices;

$L \quad$ element length;

$\left\{\mathscr{L}_{i}\right\} \quad$ standard orthogonal basis vectors spanning space $\mathfrak{P}_{n}^{r}$;

$m \quad$ dimension of the $\mathfrak{B}$ subspace;

$M_{0} \quad$ applied end moment;

$N_{i},[N]$ shape function and shape function matrix respectively;

$P \quad$ applied nodal load;

$\mathfrak{P}_{n}^{r} \quad$ polynomial function space;

$\{q\} \quad$ strain error vector;

$\left\{R^{e}\right\} \quad$ nodal reaction vector;

$\mathfrak{R}^{r} \quad$ real number space of $r$-dimensions;

$\left\{u_{i}\right\} \quad$ orthogonal basis vectors spanning the $\mathfrak{B}$ subspace;

$w \quad$ transverse displacement;

$x \quad$ coordinate of a point with element centre as origin;

$\left\{\alpha_{i}\right\} \quad$ coefficient vector of $r$-rows for the space $\mathfrak{P}_{n}^{r}$; 
$\left\{\delta^{e}\right\} \quad$ nodal displacement coordinate vector;

$\{\varepsilon\} \quad$ analytical strain vector;

$\{\bar{\varepsilon}\},\{\bar{\sigma}\} \quad$ field-inconsistent finite element strain and stress resultant vectors respectively;

$\left\{\bar{\varepsilon}^{*}\right\},\left\{\bar{\sigma}^{*}\right\}$ field-consistent finite element strain and stress resultant vectors respectively;

$\mu \quad$ moment load per unit length;

$\rho \quad$ transverse load per unit length;

$\{\rho\} \quad$ load intensity vector for element;

$\theta \quad$ rotation of section normal to beam axis;

$\xi \quad$ non-dimensional coordinate.

\section{References}

Carpenter N, Belytschko T, Stolarski H 1986 Locking and shear scaling factors in $C^{0}$ bending elements. Comput. Struct. 22: 39-52

Edwards L H, Penny D E 1988 Elementary linear algebra (Englewood Cliffs, NJ: Prentice-Hall)

Mukherjee S, Prathap G 2001 Analysis of shear locking in Timoshenko beam elements using the function space approach. Commun. Numer. Methods Eng. 17: 385-393

Prathap G 1982 Reduced integration and the shear flexible beam element. Int. J. Numer. Methods Eng. 18: $195-210$

Prathap G 1987 Field-consistency and violent stress oscillations in the finite element method. Int. J. Numer. Methods Eng. 24: 2017-2033

Prathap G 1993 The finite element method in structural mechanics (Dordrecht: Kluwer Academic)

Tessler A, Hughes T J R 1983 An improved treatment of transverse shear in the Mindlin type fournode quadrilateral element. Comput. Methods Appl. Mech. Eng. 39: 311-335

Zienkiewicz O C, Taylor R L 1991 The finite element method (New York: McGraw-Hill) 